\title{
MicroRNA-503 serves an oncogenic role in retinoblastoma progression by directly targeting PTPN12
}

\author{
YANG CHENG and WEI LIU \\ Department of Ophthalmology, Union Hospital, Tongji Medical College, \\ Huazhong University of Science and Technology, Wuhan, Hubei 430022, P.R. China
}

Received August 23, 2018; Accepted April 5, 2019

DOI: $10.3892 /$ etm.2019.7795

\begin{abstract}
Numerous studies have demonstrated that microRNAs (miRNAs or miRs) are abnormally expressed in retinoblastoma $(\mathrm{RB})$. miRNAs may serve a role in oncogene or tumor-suppressor activity in RB genesis and development by modulating various biological processes. miRNAs therefore, may be effective therapeutic targets for miRNA-based therapy in patients with RB. Recently it has been revealed that miR-503 may serve a role in various types of human cancer. However, the expression and functional roles of miR-503 are rarely reported in $\mathrm{RB}$. In the current study, the expression of miR-503 was significantly upregulated in RB tissues and cell lines. In addition, Cell Counting Kit- 8 and in vitro invasion assays were performed to assess cell proliferation and invasion, respectively. The results of the present study revealed that miR-503 inhibition impeded RB in vitro cell proliferation and invasion. Furthermore, protein tyrosine phosphatase nonreceptor type 12 (PTPN12) was demonstrated to be a direct target gene of miR-503 in RB cells. PTPN12 overexpression also led to the downregulation of miR-503 in RB cell proliferation and invasion. PTPN12 knockdown could therefore abrogate the effects of miR-503 downregulation in RB cells. In conclusion, the results demonstrated that miR-503 may serve a role in $\mathrm{RB}$ oncogenic activity progression by directly targeting PTPN12. Therefore, miR-503 may be a target for effective therapy in patients with RB.
\end{abstract}

\section{Introduction}

Retinoblastoma (RB), the most prevalent intraocular cancer, is a childhood malignant tumor derived from immature cells in the retina (1). RB accounts for $\sim 2-4 \%$ of all childhood

Correspondence to: Professor Yang Cheng, Department of Ophthalmology, Union Hospital, Tongji Medical College, Huazhong University of Science and Technology, 1277 Jiefang Road, Wuhan, Hubei 430022, P.R. China

E-mail: yangcheng_8795@126.com

Key words: retinoblastoma, microRNA-503, proliferation, invasion, protein tyrosine phosphatase nonreceptor type 12 malignancies, in which the morbidity is $\sim 1: 15,000-1: 20,000$ (2). The typical clinical symptoms of RB include strabismus, nystagmus, red eyes and blindness, which are attributed to the position of the tumor (3). Multiple factors, including genetic and epigenetic mutation, inactivation of tumor suppressors and the activation of oncogenes, have been revealed to be closely associated with the pathogenesis of RB $(4,5)$. However, the detailed mechanisms responsible for RB occurrence and development remain undetermined. Despite significant developments in RB diagnostic and treatment methods, the therapeutic outcomes for patients remains poor (6-8). Therefore, assessing the molecular mechanisms of RB formation and progression may provide the basis for their identification as promising therapeutic targets for the treatment of this aggressive disease.

Recently, microRNAs (miRNAs or miRs) have been revealed to be important in cancer research (9). These endogenous, non-coding and short RNAs are able to regulate gene expression through the binding of miRNA 'seed' regions to complementary sequences of the 3'-untranslational region (3'-UTR) of target genes, ultimately causing mRNA degradation and/or translation reduction (10). In total, 4,469 different miRNAs, including 1,881 precursor and 2,588 mature miRNAs, have been identified in the human genome (11). A growing body of evidence has revealed that miRNAs serve a crucial role within carcinogenesis and cancer progression through their effect on numerous physiological and pathological processes including cell proliferation, cycle, apoptosis, angiogenesis, differentiation, metabolism, invasion and metastasis (12-14). The aberrant expression of miRNAs has been observed in nearly all types of human cancer, including RB (15), lung cancer (16), gastric cancer (17) and thyroid cancer (18). Depending on the characteristics of their targets, miRNAs may serve an oncogenic or tumor suppressor role within the progression and development of RB $(19,20)$. However, further investigation into the detailed roles and mechanisms underlying the effects of dysregulated miRNAs in RB may reveal effective targets for use in patient therapy.

miR-503 has been reported to be dysregulated, serving crucial roles in many types of human cancer, including non-small cell lung cancer (21), hepatocellular carcinoma (22), endometrial cancer (23) and cervical cancer (24). However, the biological roles and expression patterns of miR-503 in RB have not yet been fully elucidated. In the current study, miR-503 expression in RB tissues and cell lines was detected, 
and the role of miR-503 in the development of RB was also determined. In addition, the mechanisms underlying the activity of miR-503 in RB were assessed. The results of the current study indicate that miR-503 may represent a potential therapeutic target for patients with RB.

\section{Materials and methods}

Tissue specimens. The current study was approved by the Ethics Committee of Union Hospital, Tongji Medical College, Huazhong University of Science and Technology (Hubei, China). All participants provided written informed consent for the use of their clinical tissues. RB specimens were collected from 26 patients with RB (17 males; 9 females; age range, 15-43 years) who received surgery at Union Hospital, Tongji Medical College, Huazhong University of Science and Technology (Hubei, China) between August 2015 and July 2017. A total of 8 normal retinal tissues were obtained from patients (5 males; 3 females; age range, 28-61 years) suffering from globe rupture. All patients who received radiotherapy or chemotherapy were excluded from the current study. Fresh tissues were frozen in liquid nitrogen followed by transfer to a $-80^{\circ} \mathrm{C}$ cryogenic refrigerator until further use.

Cell culture. A total of three RB cell lines (SO-RB50, Y79 and Weri-RB1) and a normal retinal pigmented epithelial cell line, ARPE-19, were purchased from the American Type Culture Collection. Cells were cultured at $37^{\circ} \mathrm{C}$ in a humidified incubator supplied with 5\% $\mathrm{CO}_{2}$. DMEM containing $10 \%$ $\mathrm{v} / \mathrm{v}$ heat-inactivated FBS, $100 \mathrm{U} / \mathrm{ml}$ penicillin and $100 \mathrm{mg} / \mathrm{ml}$ streptomycin (all, Gibco; Thermo Fisher Scientific, Inc.) was used to culture all cell lines.

Transfection assay. A miR-503 inhibitor and the corresponding negative control miRNA inhibitor (NC inhibitor) were purchased from Shanghai GenePharma Co., Ltd. The miR-503 inhibitor sequence was 5'-CUGCAGAACUGU UCCCGCUGCUA-3' and the NC inhibitor sequence was 5'-ACUACUGAGUGACAGUAGA-3'. For protein tyrosine phosphatase nonreceptor type 12 (PTPN12) silencing, small interfering RNA (siRNA) targeting PTPN12 (cat. no. siB0729143707-1-5; PTPN12 siRNA) or negative control siRNA (cat. no. siN0000002-1-5; NC siRNA) were purchased from Guangzhou Ribobio Co., Ltd. To restore PTPN12 expression, the PTPN12 overexpression plasmid pcDNA3.1-PTPN12 (pc-PTPN12) and an empty pcDNA3.1 plasmid were constructed by the Chinese Academy of Sciences. Cells were inoculated into six-well plates with a density of $8 \times 10^{5}$ cells per well one night prior to transfection at $37^{\circ} \mathrm{C}$. Transfection of 100 pmol oligonucleotide, 100 pmol siRNA or $4 \mu \mathrm{g}$ plasmid was performed using Lipofectamine 2000 (Invitrogen; Thermo Fisher Scientific, Inc.), in accordance with manufacturers protocol. The reverse transcription-quantitative (RT-q) PCR analysis was carried out at $48 \mathrm{~h}$ post-transfection. Cell Counting Kit-8 (CCK-8) and in vitro invasion assays were performed after 24 - and 48 -h incubations at $37^{\circ} \mathrm{C}$, respectively.

$R T-q P C R$. Total RNA was extracted from clinical samples and cells using a Trizol reagent (Invitrogen; Thermo Fisher Scientific, Inc.), in accordance with manufacturer's protocol.
The concentration and purity of total RNA was evaluated using NanoDrop-2000 (Thermo Fisher Scientific, Inc.).

To detect miR-503, total RNA was reverse transcribed into cDNA using a TaqMan MicroRNA Reverse Transcription kit (Applied Biosystems; Thermo Fisher Scientific, Inc.). The thermocycling conditions for reverse transcription were as follows: $16^{\circ} \mathrm{C}$ for $30 \mathrm{~min}, 42^{\circ} \mathrm{C}$ for $30 \mathrm{~min}$ and $85^{\circ} \mathrm{C}$ for $5 \mathrm{~min}$. qPCR was subsequently performed using the Bio-Rad CFX96TM Real-Time PCR System (Bio-Rad Laboratories, Inc.) with a TaqMan MicroRNA PCR kit (Applied Biosystems; Thermo Fisher Scientific, Inc.). The temperature protocol for qPCR was as follows: $50^{\circ} \mathrm{C}$ for $2 \mathrm{~min}, 95^{\circ} \mathrm{C}$ for $10 \mathrm{~min}$, and 40 cycles of denaturation at $95^{\circ} \mathrm{C}$ for $15 \mathrm{sec}$ and annealing/extension at $60^{\circ} \mathrm{C}$ for $60 \mathrm{sec}$.

For the quantification of PTPN12 expression, a PrimeScript RT Reagent kit was used for reverse transcription and synthesized cDNA was subjected to quantitative PCR using a SYBR Premix Ex Taq ${ }^{\mathrm{TM}}$ (both, Takara Biotechnology Co., Ltd.). The temperature protocol for reverse transcription was as follows: $37^{\circ} \mathrm{C}$ for $15 \mathrm{~min}$ and $85^{\circ} \mathrm{C}$ for $5 \mathrm{sec}$. The thermocycling conditions for qPCR were as follows: $5 \mathrm{~min}$ at $95^{\circ} \mathrm{C}$, followed by 40 cycles of $95^{\circ} \mathrm{C}$ for $30 \mathrm{sec}$ and $65^{\circ} \mathrm{C}$ for $45 \mathrm{sec}$.

U6 small nuclear RNA and GAPDH were employed as internal controls to normalize the relative expression of miR-503 and PTPN12, respectively. All data were analyzed using the $2^{-\Delta \Delta C q}$ method (25). The primer sequences were as follows: miR-503 forward, 5'-GCGTAGCAGCGGGAACAG T-3' and reverse, 5'-CCAGTGCGTGTCGTGGAGT-3'; U6 forward, 5'-GCTTCGGCAGCACATATACTA-3' and reverse, 5'-CGCTTCACGAATTTGCGTGTC-3'; PTPN12 forward, 5'-GCAGGAACAACACATTCAGG-3' and reverse, 5'-TCC ATTCCGATCTTACAGGTG-3'; GAPDH forward, 5'-CGG AGTCAACGGATTTGGTCGTAT-3' and reverse, 5'-AGC CTTCTCCATGGTGGTGAAGAC -3'.

CCK- 8 assay. Transfected cells were harvested $24 \mathrm{~h}$ after incubation at $37^{\circ} \mathrm{C}$ and resuspended in DMEM containing $10 \%$ FBS. A total of 3,000 transfected cells in $100 \mu \mathrm{l}$ culture medium were inoculated per well into 96-well plates. Cells were incubated at $37^{\circ} \mathrm{C}$ in an atmosphere supplied with $5 \% \mathrm{CO}_{2}$ for $0,24,48$ and $72 \mathrm{~h}$. A CCK-8 assay was then performed to assess cellular proliferation at these time points. CCK-8 solution (Dojindo Molecular Technologies, Inc.; $10 \mu \mathrm{l}$ ) was added into each well followed by incubation at $37^{\circ} \mathrm{C}$ for a further $2 \mathrm{~h}$. Absorbance at $450 \mathrm{~nm}$ was measured using a microplate reader (Bio-Rad Laboratories, Inc.).

In vitro invasion assay. The invasive ability of $\mathrm{RB}$ cells was determined using transwell apparatus pre-coated with Matrigel (each, BD Biosciences). Cells with appropriate transfection treatments were collected after $48 \mathrm{~h}$ of incubation at $37^{\circ} \mathrm{C}$ and resuspended in FBS-free DMEM. In total, $5 \times 10^{4}$ transfected cells were resuspended in FBS-free DMEM were plated into the upper compartment of transwell apparatus and bottom compartments were covered with $500 \mu$ l DMEM containing $20 \%$ FBS. After a $24-\mathrm{h}$ incubation at $37^{\circ} \mathrm{C}$, the non-invasive cells were removed with a cotton swab, while the invasive cells were fixed with $4 \%$ paraformaldehyde at room temperature for 30 min, stained with $0.5 \%$ crystal violet at room temperature for $30 \mathrm{~min}$ and air-dried. Invasive ability was assessed by 

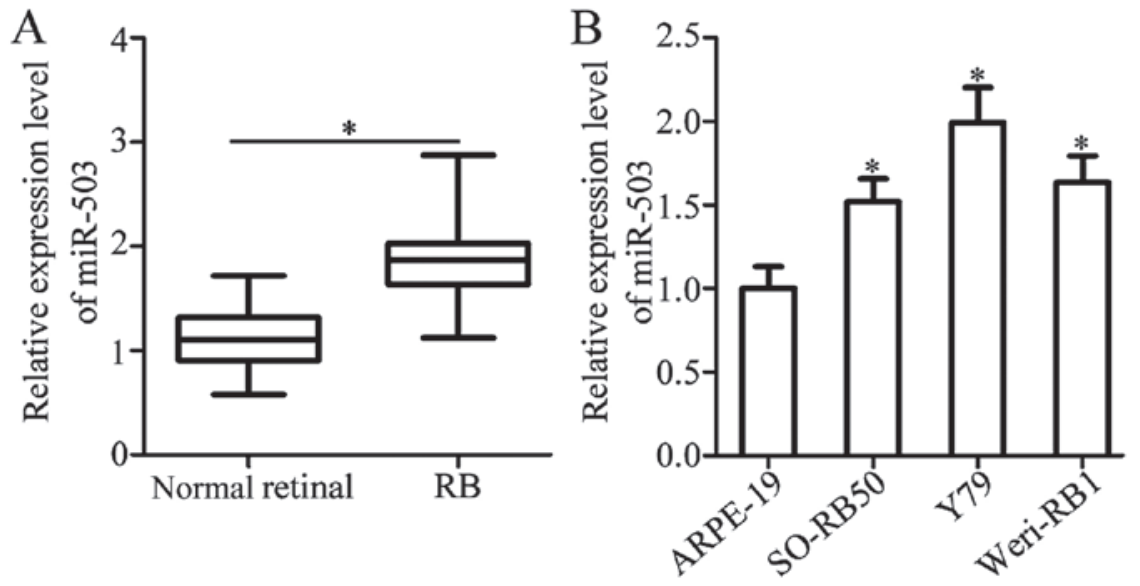

Figure 1. miR-503 is upregulated in RB tissues and cell lines. (A) Reverse transcription-quantitative PCR was used to determine miR-503 expression in RB tissues and normal retinal tissues. ${ }^{*} \mathrm{P}<0.05$ vs. normal retinal tissues. (B) The expression of miR-503 in three $\mathrm{RB}$ cell lines and a normal retinal pigmented epithelial cell line. ${ }^{*}<0.05$ vs. ARPE-19. miR-503, mRNA-503; RB, retinoblastoma.

counting the number of invasive cells in five random fields of view using a light microscope (magnification, x200; CKX41; Olympus Corporation).

Bioinformatics prediction. The putative genes of miR-503 were predicted using the miRNA target prediction software, TargetScan (http//www.targetscan.org) and miRDB (http://www.mirdb.org/).

Luciferase reporter assay. The 3'-UTR of PTPN12 containing the wild-type (wt) miR-503 binding site and its mutant (mut) 3'-UTR were chemically synthesized by Shanghai GenePharma Co., Ltd. and inserted into the pMIR-REPORT miRNA Expression Reporter vector (Ambion; Thermo Fisher Scientific, Inc.) to generate pMIR-wt-PTPN12-3'-UTR and pMIR-mut-PTPN12-3'-UTR, respectively. One night prior to transfection, cells were plated into 24-well plates with an initial density of $1.0 \times 10^{5}$ cells/well. Co-transfection of a miR-503 inhibitor or NC inhibitor and pMIR-wt-PTPN12-3'-UTR or pMIR-mut-PTPN12-3'-UTR was performed using Lipofectamine 2000 (Invitrogen; Thermo Fisher Scientific, Inc.), following the manufacturers protocol. The Dual-Luciferase ${ }^{\circledR}$ Reporter Assay system (cat. no. E1910; Promega Corporation) was used to detect luciferase activity $48 \mathrm{~h}$ after transfection. Relative luciferase activity was normalized to that of Renilla luciferase activity.

Western blot analysis. A Total Protein Extraction kit (Nanjing KeyGen Biotech Co., Ltd.) was used to isolate total cellular protein from cultured cells according to the manufacturer's protocol. The concentration of total protein was detected using a BCA Protein Quantification kit (Beyotime Institute of Biotechnology). Equivalent proteins (30 $\mu \mathrm{g} / \mathrm{lane})$ were resolved on $10 \%$ sodium dodecyl sulfate-polyacrylamide gels, transferred to PVDF membranes and then blocked with $5 \%$ fat-free milk at room temperature for $2 \mathrm{~h}$. The following primary antibodies were then added and incubated overnight at $4^{\circ} \mathrm{C}$ : Rabbit anti-human PTPN12 (1:1,000; cat. no. ab154892) and rabbit anti-human GAPDH (1:1,000; cat. no. ab181603; both, Abcam). The membranes were subsequently probed with the goat anti-rabbit horseradish peroxidase-conjugated secondary antibody (1:5,000; cat. no. ab6721; Abcam) for $1 \mathrm{~h}$ at room temperature. Specific protein bands were developed by an enhanced chemiluminescence system (EMD Millipore). GAPDH was used as a loading control. Quantity One software version 4.62 (Bio-Rad Laboratories, Inc.) was used for the quantification of protein bands.

Statistical analysis. Statistical analysis was performed using SPSS version 17.0 (SPSS, Inc.). Differences between groups were assessed using a Student's t-tests or one-way ANOVA. A Student-Newman-Keuls test was used as a post-hoc test in multiple group analyses. All data were expressed as the mean \pm standard deviation and $\mathrm{P}<0.05$ was considered to indicate a statistically significant result.

\section{Results}

Expression of miR-503 is upregulated in RB tissues and cell lines. To determine the expression status of miR-503 in RB, RT-qPCR was performed in $26 \mathrm{RB}$ and 8 normal retinal tissues. The results reveal that the expression of miR-503 in RB tissues was significantly higher compared with normal retinal tissues (Fig. 1A; $\mathrm{P}<0.05$ ). miR-503 expression in three RB cell lines (SO-RB50, Y79 and Weri-RB1) and a normal retinal pigmented epithelial cell line (ARPE-19) were subsequently determined using RT-qPCR. The significant upregulation of miR-503 was observed in all three RB cell lines compared with ARPE-19 cells (Fig. 1B; P<0.05). The results demonstrate that miR-503 is highly expressed in $\mathrm{RB}$ and that the upregulation of miR-503 may be associated with $\mathrm{RB}$ progression.

miR-503 knockdown suppresses the proliferation and invasion of $R B$ cells. To identify the specific roles of miR-503 in the development of RB, Y79 and Weri-RB1 cells with the highest relative miR-503 level among the three RB cell lines were selected for further study and transfected with a miR-503 inhibitor or NC inhibitor. RT-qPCR analysis revealed that the transfection of miR-503 inhibitor significantly decreased 
miR-503 expression in Y79 and Weri-RB1 cells (Fig. 2A; $\mathrm{P}<0.05)$. Subsequently, a CCK-8 assay was utilized to detect the proliferative ability of RB cells, which were transfected with the miR-503 inhibitor or NC inhibitor. The results demonstrated that the downregulation of miR-503 significantly decreased the proliferation of Y79 and Weri-RB1 cells compared with $\mathrm{NC}$ inhibitor treated cells after 48 and $72 \mathrm{~h}$ (Fig. 2B; $\mathrm{P}<0.05$ ). Furthermore, an in vitro invasion assay was used to investigate the effect of miR-503 downregulation on RB cell invasion. As indicated in Fig. 2C, the inhibition of miR-503 led to a marked decrease in the invasive capacity of Y79 and Weri-RB1 cells $(\mathrm{P}<0.05)$. The results of the present study indicate that miR-503 may serve an oncogenic role in RB.

PTPN12 is a direct target gene of miR-503 in RB cells. To assess the underlying mechanisms that may be responsible for the action of miR-503 in RB cells, bioinformatics analysis was performed to predict the potential targets of miR-503. PTPN12 possessed miR-503 binding sequences in its 3'-UTR regions (Fig. 3A). PTPN12 has previously been demonstrated to serve as a tumor-suppressant in multiple types of human malignancy, so was selected for additional analysis (26-29). A luciferase reporter assay was used to verify the prediction that PTPN12 served a role in the expression of miR-503. Y79 and Weri-RB1 cells were co-transfected with a miR-503 inhibitor or an NC inhibitor and pMIR-wt-PTPN12-3'-UTR or pMIR-mut-PTPN12-3'-UTR. Luciferase activity detection at $48 \mathrm{~h}$ post-transfection revealed that the downregulation of miR-503 significantly increased the luciferase activity of the plasmid carrying wild-type miR-503 binding site in Y79 and Weri-RB1 cells (Fig. 3B; P<0.05). However, the luciferase activity in Y79 and Weri-RB1 cells co-transfected with the miR-503 inhibitor and pMIR-mut-PTPN12-3'-UTR was not altered significantly (Fig. 3B). To assess the roles of miR-503 in the regulation of PTPN12 expression, RT-qPCR and western blot analysis were performed in order to measure PTPN12 expression in Y79 and Weri-RB1 cells in response to miR-503 downregulation. The mRNA (Fig. 3C; $\mathrm{P}<0.05$ ) and protein (Fig. 3D; P<0.05) expression of PTPN12 were significantly upregulated in Y79 and Weri-RB1 cells treated with the miR-503 inhibitor. Collectively, these results indicate that PTPN12 may be a direct target gene of miR-503 in RB cells.

PTPN12 restoration phenocopies the effects of miR-503 downregulation in $R B$ cells. To further assess whether PTPN12 is a direct functional downstream target of RB cell miR-503, a series of functional assays were performed to investigate whether the effects of miR-503 downregulation in RB cells could be achieved by PTPN12 upregulation. Y79 and Weri-RB1 cells were transfected with the PTPN12 overexpression plasmid pcDNA3.1-PTPN12 (pc-PTPN12) to significantly enhance PTPN12 expression (Fig. 4A; P $<0.05$ ). CCK-8 and in vitro invasion assays revealed that resumption of PTPN12 expression significantly restricted the proliferation (Fig. 4B; $\mathrm{P}<0.05$ ) and invasion (Fig. 4C; $\mathrm{P}<0.05$ ) of $\mathrm{Y} 79$ and Weri-RB1 cells, which were similar with those induced by miR-503 downregulation. These results further demonstrate that PTPN12 is a direct target gene of miR-503 in RB cells.
PTPN12 silencing abolishes the effects of miR-503 knockdown in RB cells. Rescue experiments were performed within the current study in order to investigate whether PTPN12 was required to regulate the proliferation and invasion of $\mathrm{RB}$ cells mediated by miR-503 downregulation. Y79 and Weri-RB1 cells were co-transfected with a miR-503 inhibitor and a specific siRNA targeting PTPN12 (PTPN12 siRNA) or NC siRNA. Western blot analysis revealed that miR-503 knockdown significantly increased PTPN12 protein expression in Y79 and Weri-RB1 cells; however, the protein level of PTPN12 was recovered in Y79 and Weri-RB1 cells after co-transfection with PTPN12 siRNA (Fig. 5A; P<0.05). Similarly, PTPN12 silencing abrogated the effects of miR-503 downregulation in $\mathrm{Y} 79$ and Weri-RB1 cell proliferation (Fig. 5B; $\mathrm{P}<0.05$ ) and invasion (Fig. 5C; $\mathrm{P}<0.05$ ), as determined by CCK-8 and in vitro invasion assays, respectively. Data from the current study demonstrates that miR-503 downregulation prohibits the proliferation and invasion of RB cells, at least partly, by negatively modulating PTPN12 expression.

\section{Discussion}

A number of studies have demonstrated that miRNAs including miR-137 (30), miR-448 (31) and miR-506 (15) are abnormally expressed in RB. It is now widely accepted that miRNAs may serve roles in tumor-suppressor or oncogene activity in RB development by modulating various biological behaviors, including cell proliferation, apoptosis, angiogenesis and metastasis (32). miRNAs therefore, may be effective therapeutic targets for miRNA-based therapy in patients with $\mathrm{RB}$. In the present study, miR-503 expression in RB tissues and cell lines was detected. In addition, the detailed roles and underlying mechanisms of miR-503 in RB progression was investigated. The current study may provide novel insight into RB pathogenesis and offer a promising therapeutic target for patients with this disease.

miR-503 is downregulated in non-small cell lung cancer, and this downregulation is significantly correlated with lymphatic invasion, distant metastasis, TNM stage and tumor grade (21). Patients with non-small cell lung cancer and low miR-503 expression possess poorer clinical outcomes than patients with high miR-503 expression (21). Multivariate analysis identifies miR-503 as an independent prognostic factor for assessing prognosis in patients with non-small cell lung cancer (21). Low expression of miR-503 is exhibited in hepatocellular carcinoma (22), endometrial cancer (23), cervical cancer (24), osteosarcoma (33), gastric cancer $(34,35)$, breast cancer (36) and prostate cancer (37,38). By contrast, miR-503 is overexpressed in colorectal $(39,40)$ and oesophageal $(41)$ cancer. However, the expression of miR-503 in RB remains unclear. In the current study therefore, RT-qPCR analysis was used for the detection of miR-503 expression in RB tissues and cell lines. The results of the present study demonstrated that miR-503 is significantly upregulated in RB tissues and cell lines. miR-503 may be an attractive biomarker for the diagnosis of patients with these specific cancer types.

miR-503 serves tumor-suppressive roles in hepatocarcinogenesis and progression by affecting cell angiogenesis, cellcycle, growth, metastasis and chemotherapy sensitivity $(22,42-45)$. In non-small cell lung cancer, restoration of miR-503 expression 


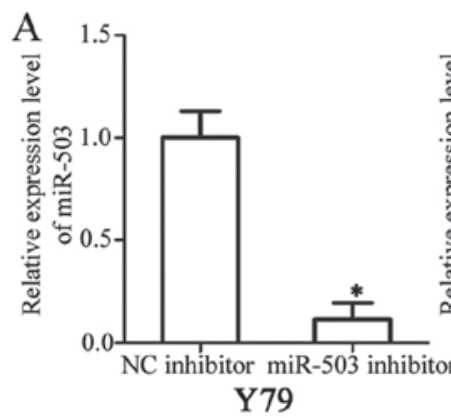

$\mathrm{C}$

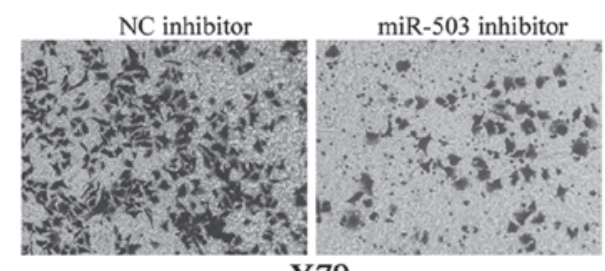

Y79

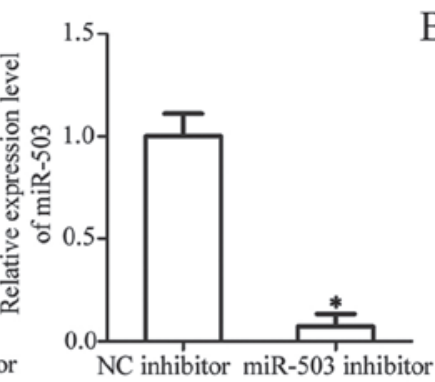

Weri-RB1
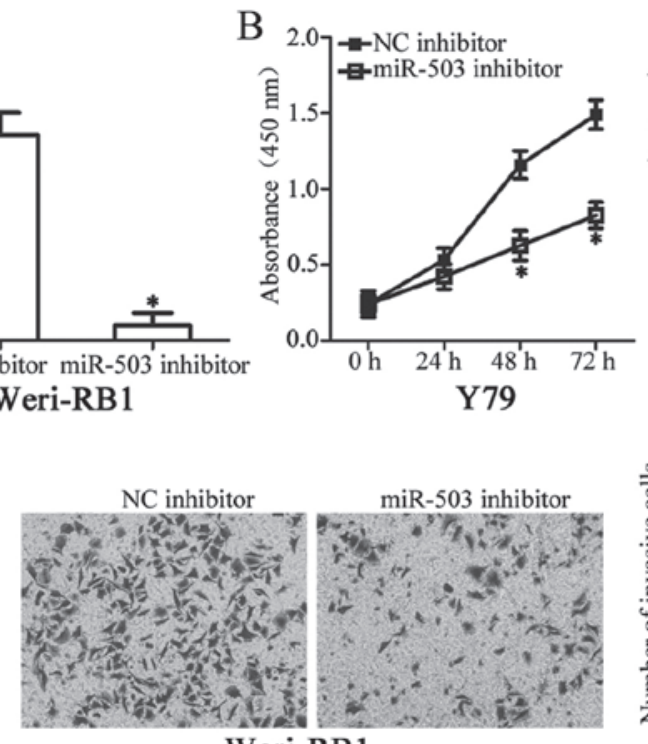

Weri-RB1
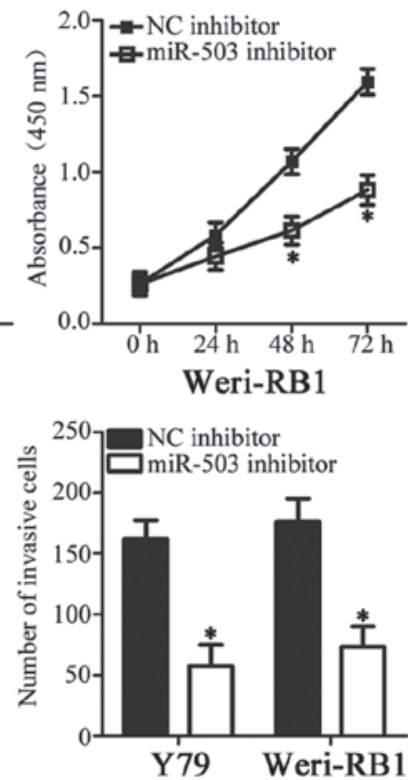

Figure 2. miR-503 knockdown inhibits the proliferation and invasion of Y79 and Weri-RB1 cells. (A) Y79 and Weri-RB1 cells transfected with a miR-503 inhibitor or an NC inhibitor were subjected to reverse transcription-quantitative PCR analysis. (B) A Cell Counting Kit-8 assay was performed to investigate the proliferation of Y79 and Weri-RB1 cells after transfection with a miR-503 inhibitor or an NC inhibitor. (C) In vitro invasion assays were performed to detect cell invasion in miR-503 inhibitor or NC inhibitor-transfected Y79 and Weri-RB1 cells. "P<0.05 vs. NC inhibitor. miR-503, mRNA-503; NC, negative control.

A

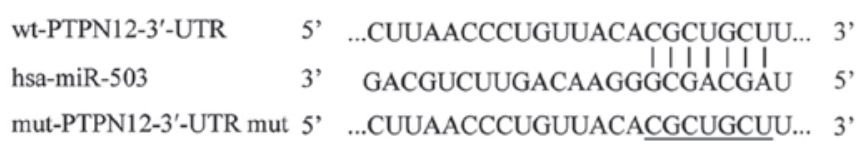

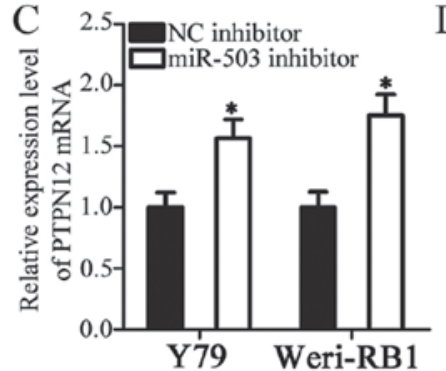

$\mathrm{B}$

D

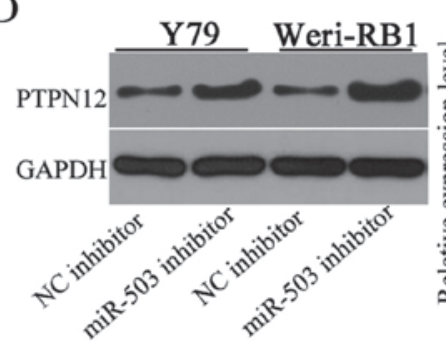

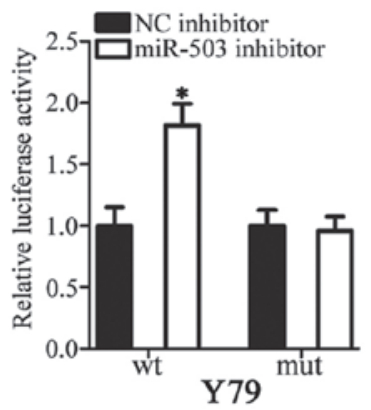
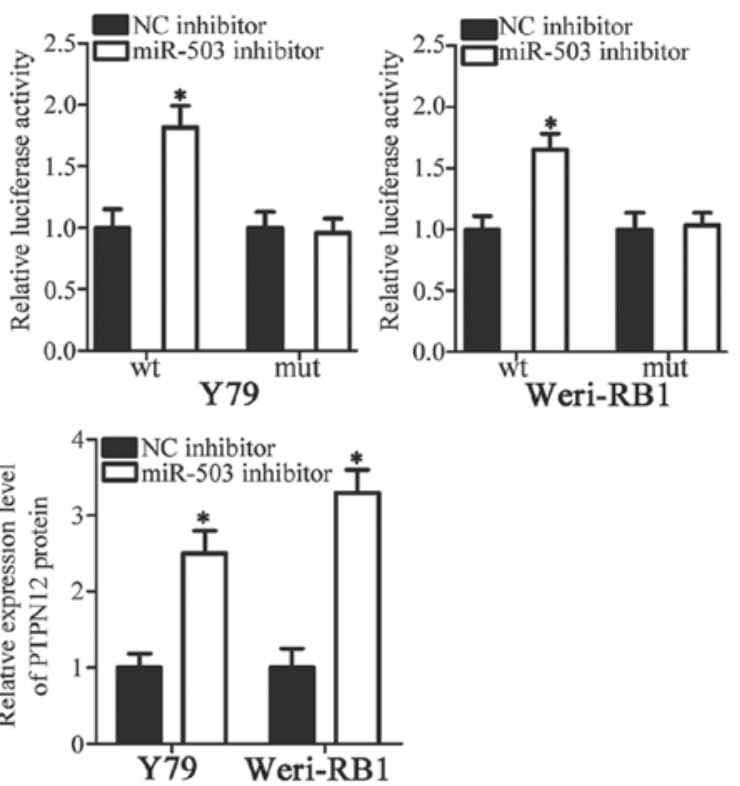

Figure 3. PTPN12 is a direct target gene of miR-503 in retinoblastoma cells. (A) PTPN12 3'-UTR and miR-503 sequences revealed using bioinformatics analysis. Wild-type and mutant miR-503 binding sites in the 3'-UTR of PTPN12 were also presented. (B) A miR-503 inhibitor or an NC inhibitor along with pMIR-wt-PTPN12-3'-UTR or pMIR-mut-PTPN12-3'-UTR was co-transfected into Y79 and Weri-RB1 cells. Following 48 h incubation, luciferase reporter assays were applied to measure the relative luciferase activity. (C) Reverse transcription-quantitative PCR and (D) western blot analysis were performed to detect PTPN12 mRNA and protein levels in Y79 and Weri-RB1 cells transfected with a miR-503 inhibitor or an NC inhibitor. ${ }^{*}<0.05$ vs. NC inhibitor. PTPN12, protein tyrosine phosphatase nonreceptor type 12; miR-504, mRNA-504; UTR, untranslated region; wt, wild-type; mut, mutant; NC, negative control.

inhibits cell proliferation and metastasis in vitro and in vivo and improves chemosensitivity to cisplatin $(46,47)$. In endometrial cancer, miR-503 upregulation attenuates cell viability, colon formation ability and cell-cycle status in vitro (23). In gastric cancer, miR-503 re-expression restricts cell proliferation, migration, invasion, epithelial-to-mesenchymal transition and cisplatin resistance $(34,35)$. In prostate cancer, resumption of miR-503 expression suppresses cell colony formation in vitro; decreases tumor growth and metastasis in vitro and in vivo $(37,38)$. In contrast, miR-503 serves as an oncogene in colorectal $(39,40)$ and oesophageal (41) cancer and participates in the regulation of biological behaviors associated with tumorigenesis and tumor development. However, the specific roles of miR-503 in RB development remain largely unknown. In the current study, CCK-8 and in vitro invasion assays were used to investigate the effects of miR-503 underexpression in 
A

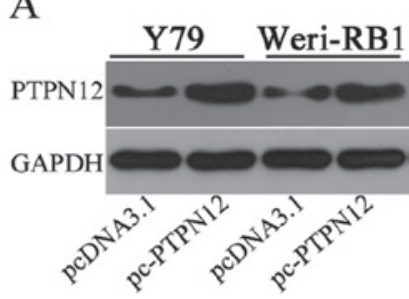

$\mathrm{C}$

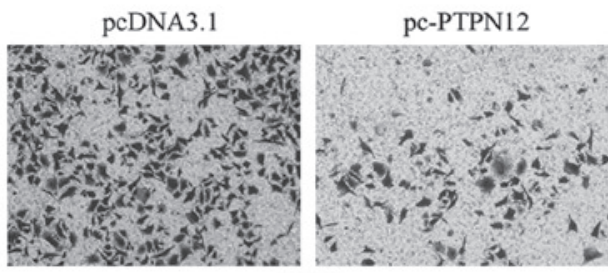

Y79
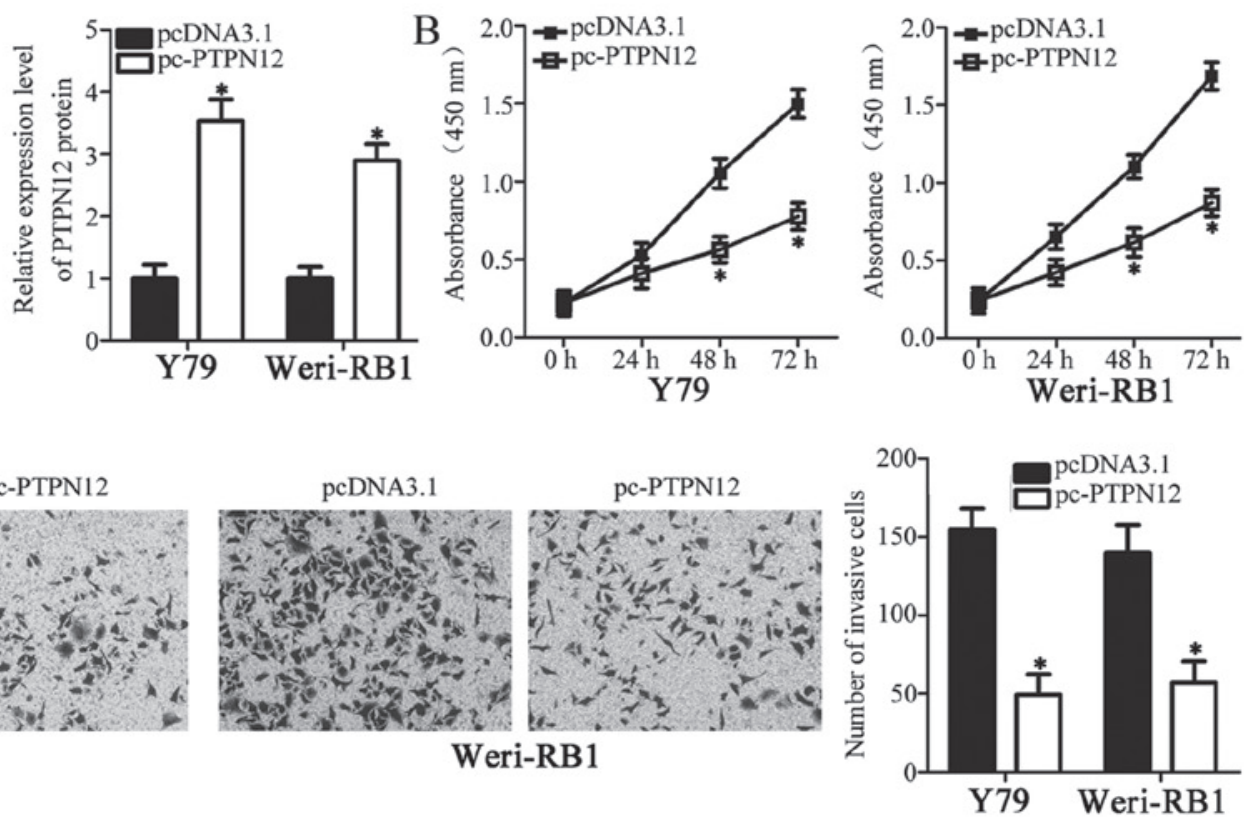

Figure 4. Upregulation of PTPN12 phenocopies the effects of miR-503 downregulation in retinoblastoma cells. Y79 and Weri-RB1 cells were treated with the PTPN12 overexpression plasmid pcDNA3.1-PTPN12 (pc-PTPN12) or empty pcDNA3.1 plasmid. (A) Protein levels of PTPN12 were determined using western blot analysis. (B) Cell proliferation was detected in Y79 and Weri-RB1 cells following transfection with pc-PTPN12 or pcDNA3.1 using a Cell Counting Kit-8 assay. (C) In vitro invasion assays were used to examine the effect of PTPN12 overexpression in the transfected Y79 and Weri-RB1 cells. "P<0.05 vs. pcDNA3.1. PTPN12, protein tyrosine phosphatase nonreceptor type 12; miR-503, mRNA-503.

A

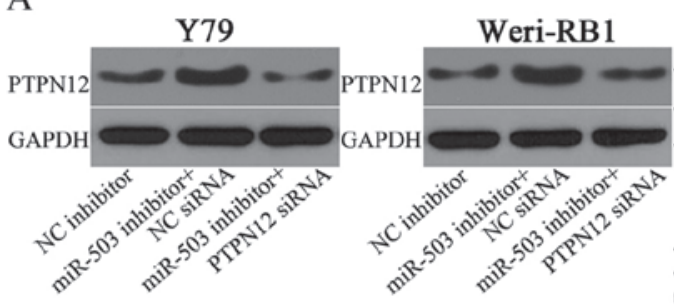

C

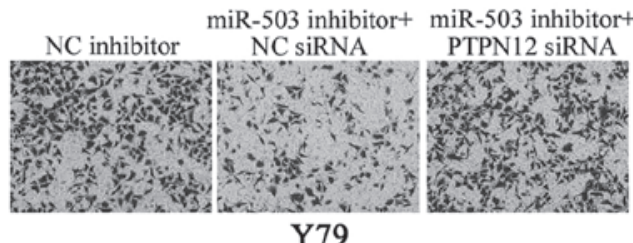

Y79

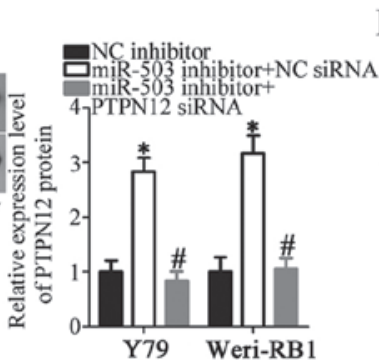

$\mathrm{B} \quad \boldsymbol{\mathrm { N } C \text { inhibitor }}$ -
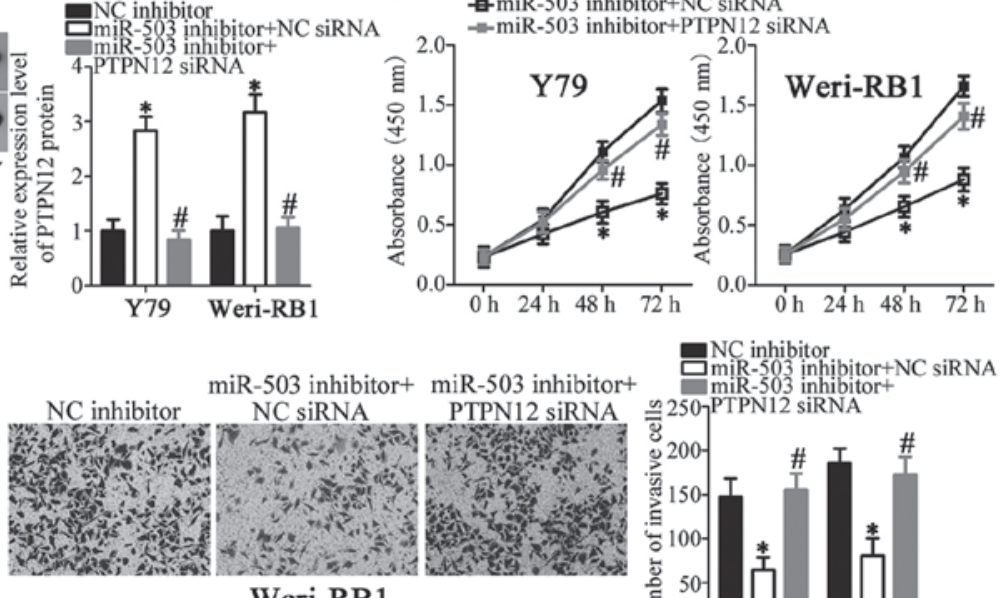

Weri-RB1

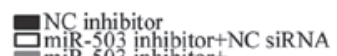

mik-503 inhibitor+

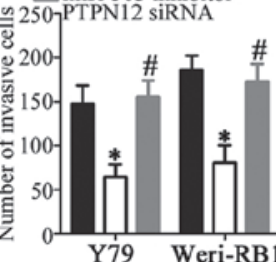

Figure 5. PTPN12 silencing rescues the effects of miR-503 downregulation in Y79 and Weri-RB1 cells. miR-503 inhibitor was co-transfected with PTPN12 siRNA or NC siRNA into Y79 and Weri-RB1 cells. (A) Transfected cells were subjected to western blot analysis for the determination of PTPN12 protein expression. (B) Cell Counting Kit-8 and (C) in vitro invasion assays were performed to observe the proliferation and invasion of Y79 and Weri-RB1 cells with different transfection treatments. ${ }^{*} \mathrm{P}<0.05$ vs. NC inhibitor. ${ }^{~} \mathrm{P}<0.05$ vs. miR-503 inhibitor + NC siRNA. PTPN12, miR-503, mRNA-503; siRNA, small interfering RNA; NC, negative control.

$\mathrm{RB}$ cell proliferation and invasion, respectively. The results of the present study demonstrated that the downregulation of miR-503 impedes the proliferative and invasive abilities of RB cells, indicating that miR-503 may be a potential therapeutic target for the anticancer therapy of patients with these human malignancies types.

Various genes have previously been identified as direct targets of miR-503, including fibroblast growth factor 2 (22), vascular endothelial growth factor A (22), cyclin D3 (42), transcription factor E2F3 (42) and protein arginine $\mathrm{N}$-methyltransferase 1 (43). In the current study, PTPN12 was demonstrated to be a direct target gene of miR-503 in RB. PTPN12, is a member of the Protein tyrosine phosphatases family (48) and is frequently downregulated in several types of human cancer, including nasopharyngeal carcinoma (28), ovarian cancer (49), breast cancer (50) and hepatocellular carcinoma (51). PTPN12 serves as a tumor-suppressor in cancer initiation and progression by regulating a wide range 
of biological processes, including cell proliferation, apoptosis, migration, invasion, metastasis and chemotherapeutic resistance (26-29). The current study demonstrated that PTPN12 upregulation inhibits the proliferation and invasion of RB cells and that the inhibition of miR-503 directly targets PTPN12, suppressing the progression of RB. Therefore, miR-503 knockdown or the restoration of PTPN12 expression may be potential therapeutic techniques for patients with RB.

In conclusion, the results of the current study revealed that miR-503 was significantly upregulated in human RB tissues and cell lines. The downregulation of miR-503 inhibited the proliferation and invasion of RB cells by directly binding to the 3'-UTR of PTPN12 and negatively regulating its expression. Due to this, miR-503 may provide further insight into RB development and offer a valuable therapeutic target for improving the outcomes of patients with RB. The current study included two limitations. Firstly, the correlation between clinical factors and miR-503 expression in patients with RB was not investigated. Secondly, the effects miR-503 overexpression RB cells were not examined. These limitations should be resolved in future study.

\section{Acknowledgements}

Not applicable.

\section{Funding}

No funding was received.

\section{Availability of data and materials}

The datasets used and/or analyzed during the present study are available from the corresponding author on reasonable request.

\section{Authors' contributions}

YC and WL designed the research. YC performed RT-qPCR, CCK-8 and in vitro invasion assays. WL performed western blot analysis, luciferase reporter assay and statistical analysis. All authors read and approved the final draft.

\section{Ethics approval and consent to participate}

The present study was approved by the Ethics Committee of Union Hospital, Tongji Medical College, Huazhong of Science and Technology (Hubei, China), and was performed in accordance with the Declaration of Helsinki and the guidelines of the Ethics Committee of Union Hospital, Tongji Medical College, Huazhong University of Science and Technology. Written informed consent was obtained from all patients for the use of their clinical tissues.

\section{Patient consent for publication}

Patient consent for publication was obtained.

\section{Competing interests}

The authors declare that they have no competing interests.

\section{References}

1. Dimaras H, Kimani K, Dimba EA, Gronsdahl P, White A, Chan HS and Gallie BL: Retinoblastoma. Lancet 379: 1436-1446, 2012.

2. Broaddus E, Topham A and Singh AD: Incidence of retinoblastoma in the USA: 1975-2004. Br J Ophthalmol 93: 21-23, 2009.

3. Abramson DH, Beaverson K, Sangani P, Vora RA, Lee TC, Hochberg HM, Kirszrot J and Ranjithan M: Screening for retinoblastoma: Presenting signs as prognosticators of patient and ocular survival. Pediatrics 112: 1248-1255, 2003.

4. Beta M, Venkatesan N, Vasudevan M, Vetrivel U, Khetan V and Krishnakumar S: Identification and insilico analysis of retinoblastoma serum microRNA profile and gene targets towards prediction of novel serum biomarkers. Bioinform Biol Insights 7: 21-34, 2013.

5. Wang J, Wang X, Wu G, Hou D and Hu Q: MiR-365b-3p, down-regulated in retinoblastoma, regulates cell cycle progression and apoptosis of human retinoblastoma cells by targeting PAX6. FEBS Lett 587: 1779-1786, 2013.

6. Jabbour P, Chalouhi N, Tjoumakaris S, Gonzalez LF, Dumont AS, Chitale R, Rosenwasser R, Bianciotto CG and Shields C: Pearls and pitfalls of intraarterial chemotherapy for retinoblastoma. J Neurosurg Pediatr 10: 175-181, 2012.

7. Kaliki S, Shields CL, Rojanaporn D, Al-Dahmash S, McLaughlin JP, Shields JA and Eagle RC Jr: High-risk retinoblastoma based on international classification of retinoblastoma: Analysis of 519 enucleated eyes. Ophthalmology 120: 997-1003, 2013.

8. Canturk S, Qaddoumi I, Khetan V, Ma Z, Furmanchuk A, Antoneli CB, Sultan I, Kebudi R, Sharma T, Rodriguez-Galindo C, et al: Survival of retinoblastoma in less-developed countries impact of socioeconomic and health-related indicators. Br J Ophthalmol 94: 1432-1436, 2010.

9. Lin S and Gregory RI: MicroRNA biogenesis pathways in cancer. Nat Rev Cancer 15: 321-333, 2015.

10. Bagnyukova TV, Pogribny IP and Chekhun VF: MicroRNAs in normal and cancer cells: A new class of gene expression regulators. Exp Oncol 28: 263-269, 2006.

11. D'Angelo B, Benedetti E, Cimini A and Giordano A: MicroRNAs: A puzzling tool in cancer diagnostics and therapy. Anticancer Res 36: 5571-5575, 2016.

12. Ambros V: The functions of animal microR NAs. Nature 431: 350-355, 2004.

13. Kloosterman WP and Plasterk RH: The diverse functions of microRNAs in animal development and disease. Dev Cell 11: 441-450, 2006.

14. Bartel DP: MicroRNAs: Genomics, biogenesis, mechanism, and function. Cell 116: 281-297, 2004.

15. Wu L, Chen Z and Xing Y: MiR-506-3p inhibits cell proliferation, induces cell cycle arrest and apoptosis in retinoblastoma by directly targeting NEK6. Cell Biol Int, 2018.

16. Jin RH, Yu DJ and Zhong M: MiR-1269a acts as an onco-miRNA in non-small cell lung cancer via down-regulating SOX6. Eur Rev Med Pharmacol Sci 22: 4888-4897, 2018.

17. Liu F, Hu H, Zhao J, Zhang Z, Ai X, Tang L and Xie L: miR-124-3p acts as a potential marker and suppresses tumor growth in gastric cancer. Biomed Rep 9: 147-155, 2018.

18. Huang Y, Yu S, Cao S, Yin Y, Hong S, Guan H, Li Y and Xiao H: MicroRNA-222 promotes invasion and metastasis of papillary thyroid cancer through targeting protein phosphatase 2 regulatory subunit B alpha expression. Thyroid 28: 1162-1173, 2018.

19. Li J and You X: MicroRNA-758 inhibits malignant progression of retinoblastoma by directly targeting PAX6. Oncol Rep 40: 1777-1786, 2018.

20. Yang L, Wei N, Wang L, Wang X and Liu QH: miR-498 promotes cell proliferation and inhibits cell apoptosis in retinoblastoma by directly targeting CCPG1. Childs Nerv Syst 34: 417-422, 2018.

21. Liu L, Qu W and Zhong Z: Down-regulation of miR-503 expression predicate advanced mythological features and poor prognosis in patients with NSCLC. Int J Clin Exp Pathol 8: 5609-5613, 2015.

22. Zhou B, MaR, Si W, Li S, Xu Y, Tu X and Wang Q: MicroRNA-503 targets FGF2 and VEGFA and inhibits tumor angiogenesis and growth. Cancer Lett 333: 159-169, 2013.

23. Xu YY, Wu HJ, Ma HD, Xu LP, Huo Y and Yin LR: MicroRNA-503 suppresses proliferation and cell-cycle progression of endometrioid endometrial cancer by negatively regulating cyclin D1. FEBS J 280: 3768-3779, 2013. 
24. Yin ZL, Wang YL, Ge SF, Guo TT, Wang L, Zheng XM and Liu J: Reduced expression of miR-503 is associated with poor prognosis in cervical cancer. Eur Rev Med Pharmacol Sci 19: 4081-4085, 2015.

25. Livak KJ and Schmittgen TD: Analysis of relative gene expression data using real-time quantitative PCR and the 2(-Delta Delta C(T)) method. Methods 25: 402-408, 2001.

26. Su Z, Tian H, Song HQ, Zhang R, Deng AM and Liu HW: PTPN12 inhibits oral squamous epithelial carcinoma cell proliferation and invasion and can be used as a prognostic marker. Med Oncol 30: 618, 2013

27. Villa-Moruzzi E: PTPN12 controls PTEN and the AKT signalling to FAK and HER2 in migrating ovarian cancer cells. Mol Cell Biochem 375: 151-157, 2013.

28. Lin Q, Wang H, Lin X, Zhang W, Huang S and Zheng Y: PTPN12 affects nasopharyngeal carcinoma cell proliferation and migration through regulating EGFR. Cancer Biother Radiopharm 33: 60-64, 2018

29. Wang YY, Liu H, Mao XY, Jin F, Ma B, Jiang JY and Cao Y: Identifying the role of PTPN12 expression in predicting the efficacy of capecitabine to neoadjuvant chemotherapy in breast cancer treatment. Eur Rev Med Pharmacol Sci 20: 3400-3409, 2016.

30. Zhang J, He J and Zhang L: The down-regulation of microRNA-137 contributes to the up-regulation of retinoblastoma cell proliferation and invasion by regulating COX-2/PGE2 signaling. Biomed Pharmacother 106: 35-42, 2018.

31. Wu S, Ai N, Liu Q and Zhang J: MicroRNA448 inhibits the progression of retinoblastoma by directly targeting ROCK1 and regulating PI3K/AKT signalling pathway. Oncol Rep 39: 2402-2412, 2018.

32. Golabchi K, Soleimani-Jelodar R, Aghadoost N, Momeni F, Moridikia A, Nahand JS, Masoudifar A, Razmjoo $\mathrm{H}$ and Mirzaei H: MicroRNAs in retinoblastoma: Potential diagnostic and therapeutic biomarkers. J Cell Physiol 233: 3016-3023, 2018

33. Chong Y, Zhang J, Guo X, Li G, Zhang S, Li C, Jiao Z and Shao M: MicroRNA-503 acts as a tumor suppressor in osteosarcoma by targeting L1CAM. PLoS One 9: e114585, 2014.

34. Peng Y, Liu YM, Li LC, Wang LL and Wu XL: microRNA-503 inhibits gastric cancer cell growth and epithelial-to-mesenchyma transition. Oncol Lett 7: 1233-1238, 2014.

35. Wang T, Ge G, Ding Y, Zhou X, Huang Z, Zhu W, Shu Y and Liu P: MiR-503 regulates cisplatin resistance of human gastric cancer cell lines by targeting IGF1R and BCL2. Chin Med J (Engl) 127: 2357-2362, 2014.

36. Long J, Ou C, Xia H, Zhu Y and Liu D: MiR-503 inhibited cell proliferation of human breast cancer cells by suppressing CCND1 expression. Tumour Biol 36: 8697-8702, 2015.

37. Guo J, Liu X and Wang M: miR-503 suppresses tumor cell proliferation and metastasis by directly targeting RNF31 in prostate cancer. Biochem Biophys Res Commun 464: 1302-1308, 2015.

38. Chi Y, Ding F, Zhang W and Du L: microRNA-503 suppresses the migration, proliferation and colony formation of prostate cancer cells by targeting tumor protein D52 like 2. Exp Ther Med 15: 473-478, 2018
39. Noguchi T, Toiyama Y, Kitajima T, Imaoka H, Hiro J, Saigusa S, Tanaka K, Inoue Y, Mohri Y, Toden S and Kusunoki M: miRNA-503 promotes tumor progression and is associated with early recurrence and poor prognosis in human colorectal cancer. Oncology 90: 221-231, 2016.

40. Li L, Zhang X, Yi Z, Liang X, Yin W and Li S: MiR-503 promotes the migration and invasion of colorectal cancer cells by regulating PDCD4. J BUON 23: 579-586, 2018.

41. Ide S, Toiyama Y, Shimura T, Kawamura M, Yasuda H, Saigusa S, Ohi M, Tanaka K, Mohri Y and Kusunoki M: MicroRNA-503 promotes tumor progression and acts as a novel biomarker for prognosis in oesophageal cancer. Anticancer Res 35: 1447-1451, 2015.

42. Xiao F, Zhang W, Chen L, Chen F, Xie H, Xing C, Yu X, Ding S, Chen K, Guo H, et al: MicroRNA-503 inhibits the G1/S transition by downregulating cyclin D3 and E2F3 in hepatocellular carcinoma. J Transl Med 11: 195, 2013.

43. Li B, Liu L, Li X and Wu L: miR-503 suppresses metastasis of hepatocellular carcinoma cell by targeting PRMT1. Biochem Biophys Res Commun 464: 982-987, 2015.

44. Xiao Y, Tian Q, He J, Huang M, Yang C and Gong L: MiR-503 inhibits hepatocellular carcinoma cell growth via inhibition of insulin-like growth factor 1 receptor. Onco Targets Ther 9: 3535-3544, 2016

45. Yang X, Zang J, Pan X, Yin J, Xiang Q, Yu J, Gan R and Lei X: miR-503 inhibits proliferation making human hepatocellular carcinoma cells susceptible to 5-fluorouracil by targeting EIF4E. Oncol Rep 37: 563-570, 2017

46. Yang Y, Liu L, Zhang Y, Guan H, Wu J, Zhu X, Yuan J and Li M: MiR-503 targets PI3K p85 and IKK- $\beta$ and suppresses progression of non-small cell lung cancer. Int J Cancer 135: 1531-1542, 2014.

47. Qiu T, Zhou L, Wang T, Xu J, Wang J, Chen W, Zhou X, Huang Z Zhu W, Shu Y and Liu P: miR-503 regulates the resistance of non-small cell lung cancer cells to cisplatin by targeting Bcl-2. Int J Mol Med 32: 593-598, 2013.

48. Tonks NK: Protein tyrosine phosphatases: From genes, to function, to disease. Nat Rev Mol Cell Biol 7: 833-846, 2006.

49. Liang T, Li L, Cheng Y, Ren C and Zhang G: MicroRNA-194 promotes the growth, migration and invasion of ovarian carcinoma cells by targeting protein tyrosine phosphatase nonreceptor type 12. Onco Targets Ther 9: 4307-4315, 2016.

50. Li J, Davidson D, Martins Souza C, Zhong MC, Wu N, Park M, Muller WJ and Veillette A: Loss of PTPN12 stimulates progression of ErbB2-dependent breast cancer by enhancing cell survival, migration and epithelial-to-mesenchymal transition. Mol Cell Biol 35: 4069-4082, 2015.

51. Luo RZ, Cai PQ, Li M, Fu J, Zhang ZY, Chen JW, Cao Y, Yun JP, Xie D and Cai MY: Decreased expression of PTPN12 correlates with tumor recurrence and poor survival of patients with hepatocellular carcinoma. PLoS One 9: e85592, 2014. 